\title{
An Assessment of Emergency Department Throughput and Provider Satisfaction after the Implementation of a Scribe Program
}

\author{
Brandon Allen, Ben Banapoor, Emily C. Weeks, and Thomas Payton \\ Department of Emergency Medicine, UF Health, USA \\ Correspondence should be addressed to Brandon Allen; brandonrallen@ufl.edu
}

Received 1 May 2014; Revised 17 July 2014; Accepted 10 August 2014; Published 3 September 2014

Academic Editor: Michael Blaivas

Copyright (C) 2014 Brandon Allen et al. This is an open access article distributed under the Creative Commons Attribution License, which permits unrestricted use, distribution, and reproduction in any medium, provided the original work is properly cited.

Objectives. To assess the impact of a scribe program on an academic, tertiary care facility. Methods. A retrospective analysis of emergency department (ED) data, prior to and after scribe program implementation, was used to quantitatively assess the impact of the scribe program on measures of ED throughput. An electronic survey was distributed to all emergency medicine residents and advanced practice providers to qualitatively assess the impact of the scribe program on providers. Results. Several throughput time measures were significantly lower in the postscribe group, compared to prescribe implementation, including time to disposition. The left without being seen (LWBS) decrease was not statistically significant. A total of 30 providers responded to the survey. $100 \%$ of providers indicated scribes are a valuable addition to the department and they enjoy working with scribes. $90 \%$ of providers indicated scribes increase their workplace satisfaction and quality of life. Conclusions. Through evaluation of prescribe and postscribe implementation, the postscribe time period reflects many throughput improvements not present before scribes began. Scribe Program implementation led to improved ED throughput for discharged patients with further system-wide challenges needing to be addressed for admitted patients.

\section{Introduction}

Emergency department (ED) overcrowding, defined as "a situation in which the demand for emergency services exceeds the ability to provide quality care within a reasonable time," was first described nearly 20 years ago and continues to pose a significant barrier to the current day delivery of timely emergency care [1-3]. The input-throughput-output conceptual model has become widely accepted in understanding the various causes of ED crowding [4]. Patient length of stay in the ED and the inability to move admitted patients from the ED to an inpatient setting, have been identified as potential contributing factors [5]. Poor ED throughput has been linked to the following patient and provider-related outcomes: increased morbidity and mortality, increased ED financial risk secondary to the loss or revenue from patients who left without being seen (LWBS), higher malpractice risk, and lower patient/provider satisfaction scores [4]. One major way to improve patient flow within the $\mathrm{ED}$ is to improve the quality and efficiency of medical record documentation via the use of a Scribe Program. A 2012 study demonstrated that within 6 months of implementing a Scribe Program in an academic emergency department throughput time was reduced by 23.26 minutes per visit and clinical productivity increased from 1.81 to 2.09 patients per hour [6].

The UF Health Emergency Department is a Level 1 Trauma Center that averages over 95,000 visits per year across three physically distinct sites: an adult emergency department, a pediatric emergency department, and a freestanding community emergency department. In September 2012, the UF ED implemented a rapid assessment zone (RAZ) concept to improve patient flow within the adult emergency department (AED). The implementation of the RAZ effectively reduced door to room, door to provider, and door to disposition time but not door to exit time. In addition, extended analysis showed a slight increase in the number of patients who left without being seen [7]. According to a 2013 paper published by the American Academy of Emergency 
Medicine (AAEM), in an ED with 50,000 visits per year and an average payer mix, the net revenue loss for every $1 \%$ of patients who leave without being seen (LWBS) is about $\$ 450,000$ per year [4]. Since the UF Health AED averages approximately 65,000 registered visits annually, the increase in LWBS secondary to the RAZ implementation warranted further investigation into various methods to further improve measures of AED throughput.

One such method was the implementation of an ED Scribe Program to decrease the amount of time that providers spend with documentation as well as to increase the speed and accuracy of medical charting. Scribes in the UF Health AED work in all areas of the department, including the 14-bed unit known as RAZ with 10 overflow beds into a subwaiting location, the 13-bed unit known as Core 1, and the 15-bed unit known as Core 2. The scribes are responsible for the documentation of information including the history of present illness (HPI), review of systems (ROS), physical exam (PE), lab results, and pertinent medical decision making (MDM) for the providers. A study published by the AMA and RAND Corporation in 2013 revealed that electronic medical records were a major factor contributing to physician dissatisfaction [8]. With the scribe program, physicians can focus on the care and management of the patient at the bedside with an enhanced focus on face-to-face communication versus computer tasks [9].

The aim of this study is to investigate the several measures of throughput that impact clinical productivity and to assess clinician satisfaction through the implementation of an emergency department Scribe Program.

\section{Methods}

2.1. Study Design. This study was performed at the UF Health Adult Emergency Department (ED) with proposal approval by the University of Florida Institutional Review Board (IRB). The quantitative arm of this study assessed the impact of the implementation of the Scribe Program on measures of Adult ED throughput and was conducted using retrospective data analysis. Administrative data was abstracted retrospectively from the University of Florida Decision Support Services (DSS) for the timeframe between June 1, 2012, and April 30, 2013, (prescribe) and June 1, 2013, and April 30, 2014 (postscribe). Variables compiled included daily number of registered visits, number of admitted, discharged, and left without being seen (LWBS) patients, and median time interval data for all patients, admitted patients, and discharged patients. Upon careful consideration, researchers decided not to include the month of May 2013, due to crossover and inconsistency during this month, while the Scribe Program was being implemented. Outcome measures were then compared between pre- and postscribe data. A secondary, qualitative arm of this study assessed the impact of the Scribe Program on providers and was conducted using a survey created with UF Qualtrics Online Survey Software.

2.2. Study Setting and Population. The Adult ED at UF Health is an academic institution that registered 66,514 patients in 2013, which translates to approximately 182 patients per day.
Thirty-two percent of daily patients arrive by ground or air ambulance. The admission rate is $33.1 \%$ and the vast majority of medical services are available at UF Health, so patients are rarely transferred out of our health system. During a shift, the scribe provides medical documentation services to all providers working in their designated area excluding the first year resident (PGY1). The scribe completes charting of the emergency medical record (EMR) either in the room with the provider or via dictation after encounter, but does not complete order entries. Scribes are assigned to work 10-hour shifts in various areas of the ED.

2.3. Outcome Measures. The primary aim of this study was to assess the impact of the implementation of a Scribe Program on measures of AED throughput. Throughput was assessed using the following commonly evaluated ED objective measures:

(1) door to triage time: the time elapsed from when the patient arrives in the ED until the patient is triaged;

(2) door to room time: the time elapsed from when the patient arrives in the ED until the patient arrives in the room;

(3) door to provider time: the time elapsed from when the patient arrives in the ED until the provider (physician) signs on to the patient's chart;

(4) door to disposition time: the time elapsed from when the patient arrives in the ED until the provider decides the patient's disposition;

(5) door to exit time: the time elapsed from when the patient arrives in the ED until the patient exits the ED;

(6) provider to disposition time: the time elapsed from when the provider (physician) signs on to the patient's chart until the provider decides the patient's disposition;

(7) disposition to exit time: the time elapsed from when the provider decides the patient's disposition until the patient exits the ED;

(8) The number of patients who left without being seen (LWBS).

These measures were analyzed before and after the implementation of the Scribe Program.

The secondary aim of this study was to assess the impact of the implementation of a Scribe Program on providers. An electronic, anonymous, password protected survey was constructed via the UF Qualtrics Online Survey Software. The link to the survey was administered via e-mail to all ED residents, physician's assistants (PA), and nurse practitioners (NP) individually, who were employed within the UF Health Adult Emergency Department (see Figure 1). The survey contained 17 questions which collectively assessed the qualitative impact of the implementation of the Scribe Program on providers (see Table 1). It was developed and revised by multiple ED physicians and scribes in order to ensure accuracy in assessing the aspects of impact desired. 
TABLE 1: Survey questions.

(1) Do you think scribes are a valuable addition to this department?

(2) From a shift stand point, have you seen an increase in work production/work flow while working with a scribe?

(3) Before scribes, how many hours following your shift did you spend charting?

(4) With a scribe, how many hours following your shift did you spend charting?

(5) Does the use of a scribe increase the amount of time you spend with patients?

(6) Do you enjoy working with a scribe?

(7) Overall, does the use of scribes increase your workplace satisfaction?

(8) Does the use of a scribe increase your quality of life?

(9) Does the use of a scribe increase your level of focus at work?

(10) Does the use of a scribe decrease your level of stress at work?

(11) Does the use of a scribe decrease your level of stress at home?

(12) Will the use of scribes extend your career as a physician/PA/NP?

(13) Does the use of a scribe help you remember to ask the patient questions that you otherwise might have forgotten to ask?

(14) Please discuss your thoughts about the use of scribes in RAZ

(15) Please discuss your thoughts about the use of scribes in Core

(16) Please discuss your thoughts about the use of scribes in the Trauma Bay

(17) Please discuss your thoughts of the Scribe Program as a whole

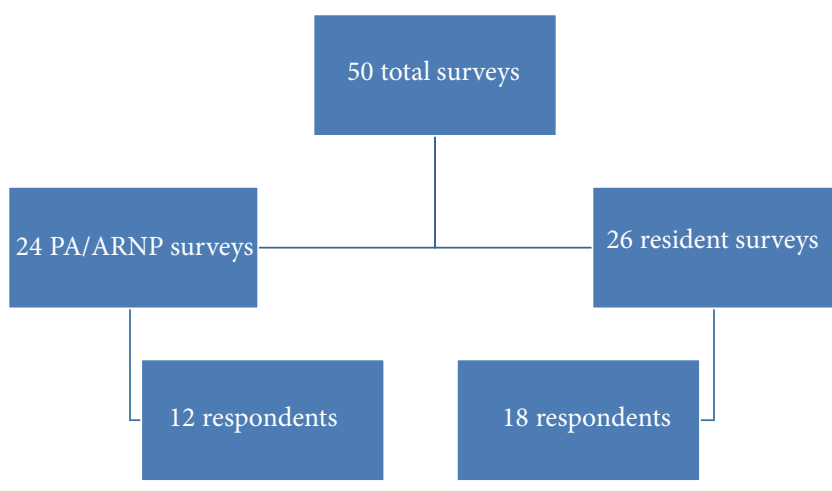

FIGURE 1: Survey demographics.

2.4. Data and Statistical Analysis. The primary aim of this study, effects of Scribe Program implementation on ED throughput, was assessed using retrospective statistical analysis methods on the pre- and postscribe data. Data was separated into prescribe (June 1, 2012, through April 30, 2013) and postscribe (June 1, 2013, through April 30, 2014) implementation cohorts, in order to assess the effects of scribe presence in the clinical areas (Table 2). In addition to the time variables previously described, time between room and disposition (the time elapsed from when the patient arrives in the room until the provider decides the patient's disposition) and time between room and exit (the time elapsed from when the patient arrives in the room until the patient exits the ED) were further assessed, in order to focus on the time that the presence of a scribe might impact facilitation of care the most. Lastly, data was assessed as an all patient group and then was further separated to look at admitted and discharged patients separately, to account for other hospital variables differing between these two groups that may obscure the impact of scribe presence. Daily medians comprised the dataset for time variables, in order to minimize effects of nonnormality and extreme length of stay outliers. $t$-tests were used to assess the significance of potential differences between the preand postscribe cohort means, considering an alpha level of 0.05 to be statistically significant. All statistical analyses were performed using SAS v.9.4 (Cary, NC).

\section{Results}

The implementation of the Scribe Program led to several statistically significant decreases in throughput variable measurements (see Table 2). Among all patients (discharged and admitted combined), time to triage, time to room, time to provider, and time to disposition were all decreased in the postscribe period, with statistical significance for time to triage $(P=0.008)$ and time to disposition $(P<0.0001)$. When separated into two cohorts (discharged patients and admitted patients), the discharged cohort reflected decreases in the postscribe implementation group in time to triage, time to room, time to provider, time to disposition, and time to exit. Statistical significance was identified in decreases for time to disposition and time to exit in this group. Among admitted patients, time to triage, time to room, and time to disposition were decreased in the postscribe implementation group, with statistically significant decreases in time to triage $(P<0.0001)$, time to room $(P=0.001)$, and time to disposition $(P<0.0001)$. The decrease in $\%$ of LWBS patients was not found to be statistically significant $(P=0.382)$. The anonymous electronic survey was sent via e-mail to 24 residents, 21 physician's assistants, and 5 nurse practitioners. There were a total of 30 survey respondents including 4 nurse practitioners, 8 physician's assistants, and 18 residents (see Table 1). $100 \%$ of all providers indicated that scribes are a valuable addition to the department and that they enjoy working with scribes. $90 \%$ of all providers indicated that scribes increase their workplace satisfaction and increase quality of life. Over $80 \%$ of all providers indicated that scribes 
TABLE 2: Measures of ED throughput (medians except mean LWBS).

\begin{tabular}{|c|c|c|c|c|}
\hline Variable & Mean prescribe (hours) & Mean postscribe (hours) & Difference (hours) & $T$-test $P$ value \\
\hline Registered visits & 181.68 & 180.69 & $\downarrow 0.99$ & 0.468 \\
\hline$\%$ admittance & 0.31 & 0.35 & $\uparrow 0.04$ & $<0.0001$ \\
\hline$\%$ LWBS & 0.05 & 0.05 & $\downarrow 0.00$ & 0.382 \\
\hline \multicolumn{5}{|c|}{ All patients (discharged and admitted) } \\
\hline Door to triage & 0.08 & 0.07 & $\downarrow 0.01$ & 0.008 \\
\hline Door to room & 0.55 & 0.54 & $\downarrow 0.01$ & 0.647 \\
\hline Door to provider & 1.34 & 1.28 & $\downarrow 0.06$ & 0.073 \\
\hline Door to disposition & 4.16 & 3.89 & $\downarrow 0.27$ & $<0.0001$ \\
\hline Door to exit & 5.62 & 5.76 & $\uparrow 0.14$ & 0.021 \\
\hline Provider to disposition & 2.82 & 2.61 & $\downarrow 0.21$ & $<0.0001$ \\
\hline Disposition to exit & 1.46 & 1.87 & $\uparrow 0.41$ & $<0.0001$ \\
\hline \multicolumn{5}{|c|}{ Discharged patients } \\
\hline Door to triage & 0.07 & 0.07 & 0.00 & 0.210 \\
\hline Door to room & 0.89 & 0.90 & $\uparrow 0.01$ & 0.782 \\
\hline Door to provider & 1.67 & 1.61 & $\downarrow 0.06$ & 0.220 \\
\hline Door to disposition & 4.57 & 4.41 & $\downarrow 0.16$ & 0.029 \\
\hline Door to exit & 5.07 & 4.89 & $\downarrow 0.18$ & 0.012 \\
\hline Provider to disposition & 2.89 & 2.80 & $\downarrow 0.09$ & 0.021 \\
\hline Disposition to exit & 0.51 & 0.48 & $\downarrow 0.03$ & 0.020 \\
\hline \multicolumn{5}{|c|}{ Admitted patients } \\
\hline Door to triage & 0.10 & 0.08 & $\downarrow 0.02$ & $<0.0001$ \\
\hline Door to room & 0.22 & 0.20 & $\downarrow 0.02$ & 0.001 \\
\hline Door to provider & 0.91 & 0.91 & 0.00 & 0.996 \\
\hline Door to disposition & 3.63 & 3.25 & $\downarrow 0.38$ & $<0.0001$ \\
\hline Door to exit & 7.61 & 8.27 & $\uparrow 0.65$ & $<0.0001$ \\
\hline Provider to disposition & 2.72 & 2.34 & $\downarrow 0.38$ & $<0.0001$ \\
\hline Disposition to exit & 3.98 & 5.01 & $\uparrow 1.03$ & $<0.0001$ \\
\hline
\end{tabular}

increase levels of focus at work and decrease levels of stress at work, and $70 \%$ of all providers indicated that scribes decrease levels of stress at home (see Table 3).

\section{Discussion}

During the 11-month period following the implementation of the ED Scribe Program the majority of measures of AED throughput were improved. Overall, most time intervals were decreased from pre- to postscribe time periods. Although some did increase, such as time to exit among all patients, we see that when broken out into discharged and admitted patients, this difference appears to be in the admitted patients and may be due to other operational factors within the hospital. Specifically, when provider to disposition and disposition to exit times are examined in the admitted group, provider to disposition time is decreased, and it is the disposition to exit time that increases, indicating that the time that scribes impact is decreased, and it is the time a patient waits to be discharged or admitted that increases. A $0.3 \%$ (5.1\% to $4.8 \%$ ) decrease in the number of patients who leave without being seen was also observed. Despite being not statistically significant, utilizing the estimates of fiscal loss by Guarisco ( $\$ 450,000$ lost for every one percent LWBS), this has the potential to capture $\$ 135,000$, in revenue, that otherwise would have been lost [4].

The increase in door to exit time of admitted patients is hypothesized to be secondary to extended holds of admitted patients. In a paper published in 2013 by Guarisco it was stated that "the major obstacle to throughput is output to the inpatient units of the hospital, most importantly due to constraints such as bed availability [10]." This could be a major contributing factor to the increase in door to exit median time of admitted patients that was observed, warranting further investigation by the institution into methods with which patient admission throughput can be improved. Moreover, scribes were primarily placed in RAZ which has an admission rate of approximately $10-15 \%$ in contrast to the Core which has an admission rate of approximately 55-60\%. The total admission rate for the AED is between 33 and 35\%, which the researchers believe underscores the significance of the discharging data rather than the admission data.

The impact of the ED Scribe Program was overwhelmingly positive on the 30 survey respondents. The researchers believe that the low amount of survey respondents (60\%) was likely secondary to some advanced practice providers working part time, as well as the emergency department's policy that PGY-1 residents are not permitted to use scribes. 
TABLE 3: Survey results.

\begin{tabular}{|c|c|c|c|}
\hline Respondent & Resident $(n=18)$ & Physician's assistant $(n=8)$ & Nurse practitioner $(n=4)$ \\
\hline \multicolumn{4}{|c|}{ (1) Do you think scribes are a valuable addition to this department? } \\
\hline Yes & $100 \%$ & $100 \%$ & $100 \%$ \\
\hline No & $0 \%$ & $0 \%$ & $0 \%$ \\
\hline \multicolumn{4}{|c|}{ (2) From a shift standpoint, have you seen an increase in work production/work flow while working with a scribe? } \\
\hline Yes, large amount & $78 \%$ & $88 \%$ & $75 \%$ \\
\hline Yes, small amount & $22 \%$ & $12 \%$ & $0 \%$ \\
\hline No & $0 \%$ & $0 \%$ & $25 \%$ \\
\hline
\end{tabular}

(3) Before scribes, how many hours following your shift did you spend charting?

$\begin{array}{llll}0-1 & 13 \% & 12 \% & 0 \% \\ 1-2 & 56 \% & 25 \% & 25 \% \\ 2+ & 31 \% & 63 \% & 75 \%\end{array}$

(4) With a scribe, how many hours following your shift did you spend charting?

\begin{tabular}{cccc}
$0-1$ & $82 \%$ & $63 \%$ & $75 \%$ \\
$1-2$ & $12 \%$ & $37 \%$ & $25 \%$ \\
$2+$ & $0 \%$ & $0 \%$ & $0 \%$ \\
\hline
\end{tabular}

(5) Does the use of a scribe increase the amount of time you spend with patients?

\begin{tabular}{|c|c|c|c|}
\hline Yes & $89 \%$ & $75 \%$ & $50 \%$ \\
\hline No & $11 \%$ & $25 \%$ & $50 \%$ \\
\hline \multicolumn{4}{|c|}{ (6) Do you enjoy working with a scribe? } \\
\hline Yes & $100 \%$ & $100 \%$ & $100 \%$ \\
\hline No & $0 \%$ & $0 \%$ & $0 \%$ \\
\hline \multicolumn{4}{|c|}{ (7) Overall, does the use of scribes increase your workplace satisfaction? } \\
\hline Yes & $67 \%$ & $88 \%$ & $100 \%$ \\
\hline No & $33 \%$ & $12 \%$ & $0 \%$ \\
\hline
\end{tabular}

(8) Does the use of a scribe increase your quality of life?

\begin{tabular}{cccc} 
Yes & $89 \%$ & $88 \%$ & $100 \%$ \\
No & $11 \%$ & $12 \%$ & $0 \%$ \\
\hline
\end{tabular}

(9) Does the use of a scribe increase your level of focus at work?

$\begin{array}{lll}\text { Yes } & 78 \% & 88 \%\end{array}$

$\begin{array}{lll}\text { No } & 22 \% & 12 \%\end{array}$

(10) Does the use of a scribe decrease your level of stress at work?

\begin{tabular}{|c|c|c|}
\hline $\mathrm{Y}$ & . & O1 \\
\hline res & 8010 & 00 \\
\hline
\end{tabular}

(11) Does the use of a scribe decrease your level of stress at home?

$\begin{array}{lll}\text { Yes } & 78 \% & 50 \%\end{array}$

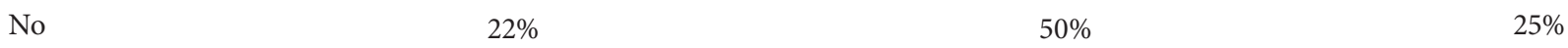

(12) Will the use of scribes extend your career as a physician/PA/NP?

Yes

$72 \% \quad 38 \% \quad 50 \%$

$\begin{array}{lll}\text { Maybe } & 28 \% & 38 \%\end{array}$

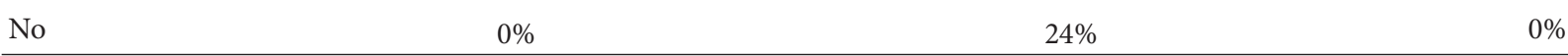

(13) Does the use of a scribe help you remember to ask the patient questions that you otherwise might have forgotten to ask?

Yes 
Approximately 18 total nonresponders to the survey met these criteria. If these providers were initially excluded, our survey completion would have been $96 \%$.

The survey concluded that $100 \%$ of all providers indicated that scribes are a valuable resource to the department and that they enjoy working with scribes. Furthermore, 90\% of all providers indicated that scribes increase their workplace satisfaction and increase quality of life, while over $80 \%$ of all providers indicated that scribes increased levels of focus at work and decreased levels of stress at work. Coinciding with this data, $70 \%$ of all providers indicated that scribes decreased levels of stress at home which one can likely attribute to decreased time documenting in the electronic medical record after shift.

Although both this study and previous studies investigating Scribe Programs have concluded that the use of Scribe Programs is monetarily significant for emergency departments, it is this study which demonstrates the intangible benefits of a Scribe Program in an academic emergency department. Increased workplace satisfaction, decreased levels of stress at home, decreased levels of stress at work, and increased levels of focus at work for the strong majority of emergency medicine residents and advanced practice providers are all intangible benefits from the implementation of a Scribe Program in an academic emergency department which cannot be accounted for monetarily.

It is also of importance to note that $63 \%$ of all provider types indicated that the use of scribes will likely extend their careers. The use of scribes allows providers to practice medicine without the barriers of documentation which they face daily. One UF Health emergency medicine resident responded to a survey question asking residents to discuss their thoughts of the Scribe Program overall: "Scribes allow me to be a doctor."

\section{Limitations}

This study involved a retrospective data analysis which introduces selection bias. In addition, the PA and NP providers primarily work in the RAZ area, which has historically had more extensive scribe coverage, although the Scribe Program has expanded in other ED areas more recently. The AED residents primarily work in the Core but still utilize scribes services in RAZ when a scribe is available [11]. During the data collection period, our Scribe Program was expanding, meaning new hires were being trained which could have influenced the quantity and quality of scribe charting. As scribes become more experienced with charting they will naturally become faster in their documentation which could ultimately further improve AED throughput.

During the study period, other measures were also put into place to improve the overall efficiency of the AED, such as improved clinical guidelines and policies, efforts to enhance communications and working relationships with admitting and consulting medical services, and better EMR support tools. However, implementation of the Scribe Program was considered the most important departmental strategy in improving overall operational efficiency and was certainly the program with the highest level of personnel and financial resources assigned to it. Therefore, the researchers do believe that the demonstrated improvements in operational metrics are mostly attributable to the scribe program.

This study was conducted within an academic institution. Further experimentation needs to be conducted in order to assess if the findings generated from our Scribe Program can be generalized to other academic and nonacademic emergency departments. Additionally, scribes within our AED are assigned to one area of the ED and work with multiple providers, whereas most other models assign each scribe to an individual provider for the entire duration of the shift.

\section{Conclusions}

Implementation of a Scribe Program at our tertiary, academic emergency department, has led to statistically significant improvements in basic ED throughput measures, including door to disposition time for all patients, door to provider time for discharged patients, door to exit time for discharged patients, and door to room time for admitted patients. Additionally, a trend towards a decreased number of patients who leave without being seen was found, although this measure was not statistically significant.

Based on survey data obtained from ED providers, having a Scribe Program also leads to overall increased job satisfaction, which in turn would be very beneficial from the perspective of minimizing provider turnover and attrition. This is important when considering the costs associated with hiring and training new providers to replace those lost due to job dissatisfaction. Employee wellness is clearly enhanced by having a Scribe Program.

For emergency department directors and administrators considering the implementation of such a program, it is very possible to conduct an analysis of the cost of the program compared to the projected costs of hiring and training new providers to replace dissatisfied personnel, as well as the costs associated with inefficient ED bed turnover and patients who leave without receiving medical care. The data presented in this study may help in this analysis.

\section{Conflict of Interests}

The authors declare that there is no conflict of interests regarding the publication of this paper.

\section{References}

[1] Canadian Association of Emergency Physicians and the National Emergency Nurses Association, "Access to acute care in the setting of emergency department overcrowding," Canadian Journal of Emergency Medicine, vol. 3, pp. 82-84, 2003.

[2] G. Dickinson, "Emergency department overcrowding," Canadian Medical Association Journal, vol. 140, no. 3, pp. 270-271, 1989.

[3] E. J. Gallagher and S. G. Lynn, “The etiology of medical gridlock: causes of emergency department overcrowding in New York city," Journal of Emergency Medicine, vol. 8, no. 6, pp. 785-790, 1990. 
[4] G. Joseph, "Cracking the code: fixing the crowded emergency department, part 1-building the burning platform," Common Sense, vol. 20, no. 5, pp. 18-20, 2013.

[5] B. R. Asplin, D. J. Magid, K. V. Rhodes, L. I. Solberg, N. Lurie, and C. A. Camargo Jr., "A conceptual model of emergency department crowding," Annals of Emergency Medicine, vol. 42, no. 2, pp. 173-180, 2003.

[6] J. S. Marshall, C. M. Verdick, M. S. Tanaka et al., "296 implementation of medical scribes in an Academic Emergency Department: effect on Emergency Department throughput, clinical productivity, and emergency physician professional fees," Annals of Emergency Medicine, vol. 60, no. 4, supplement, p. S105, 2012.

[7] P. Thomas, T. Ison, M. Falgiani et al., "Emergency department implementation of the rapid assessment zone," in Proceedings of the UFHealth Annual Quality Symposium and Retreat, Poster Presentation, July 2013.

[8] W. F. Mark, G. Peggy, R. Kristin et al., Factors Affecting Physician Professional Satisfaction and Their Implications for Patient Care, Health Systems, and Health Policy, RAND, 2014.

[9] “Scribes, EMR Please Docs, save \$600,000," ED Management, 2009.

[10] J. Guarisco, "Cracking the code: fixing the crowded emergency department, part 2-creating the analytic model," Common Sense, vol. 20, no. 6, pp. 23-25, 2013.

[11] R. Arya, D. M. Salovich, P. Ohman-Strickland, and M. A. Merlin, "Impact of scribes on performance indicators in the emergency department," Academic Emergency Medicine, vol. 17, no. 5, pp. 490-494, 2010. 


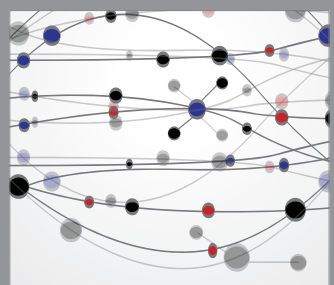

The Scientific World Journal
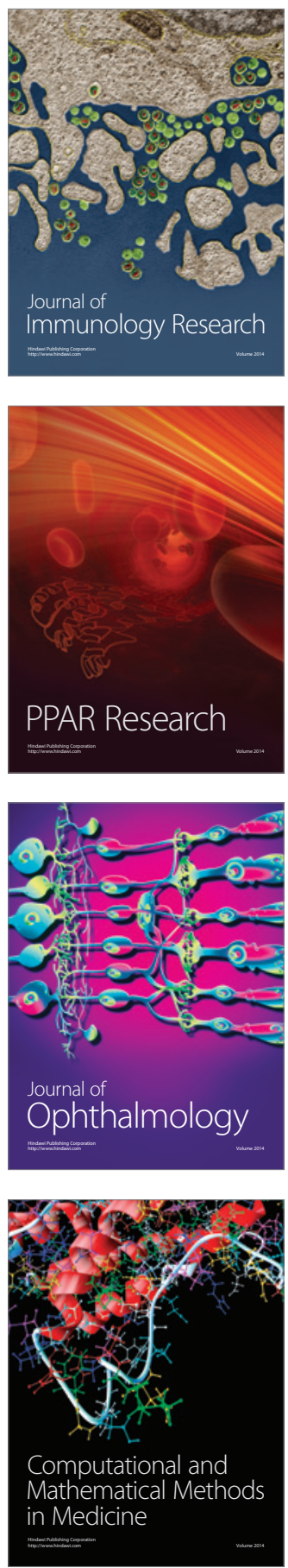

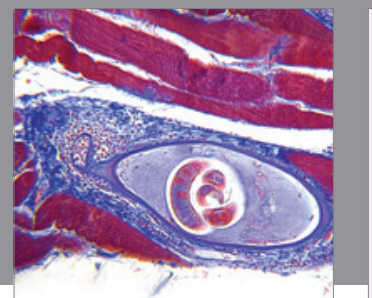

Gastroenterology

Research and Practice
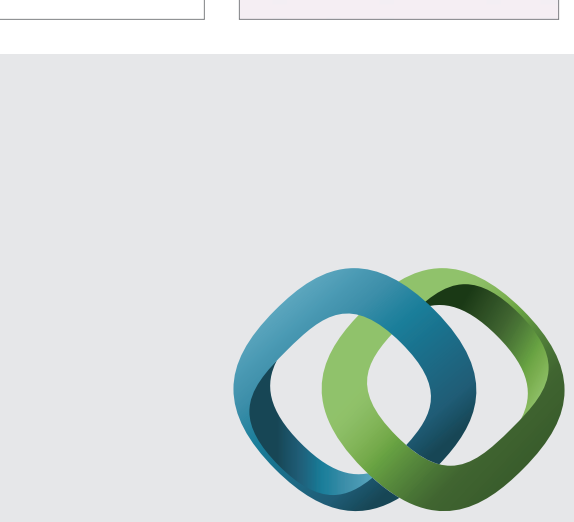

\section{Hindawi}

Submit your manuscripts at

http://www.hindawi.com
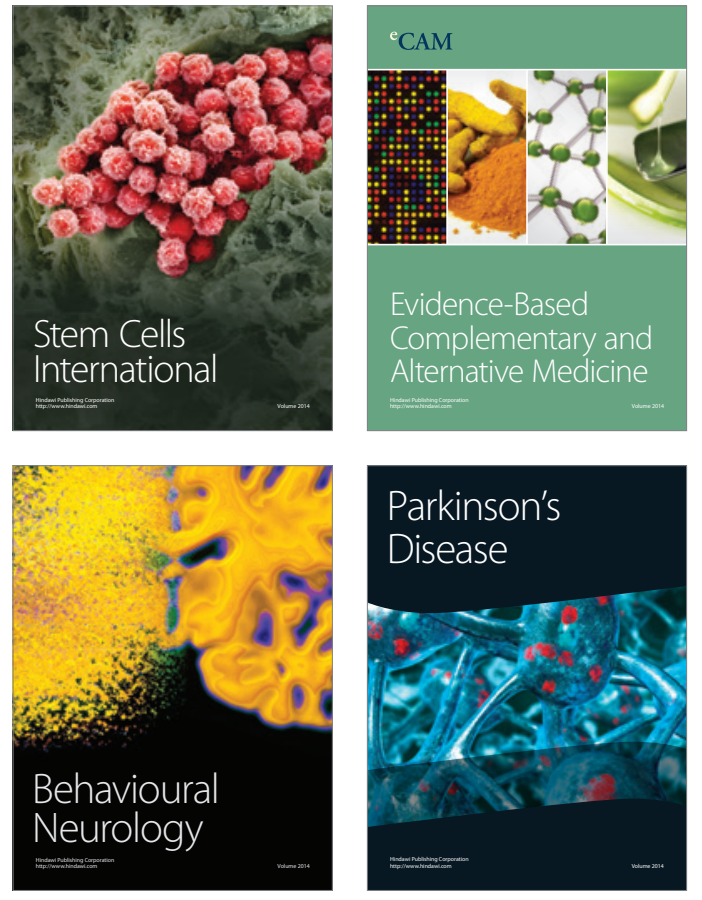
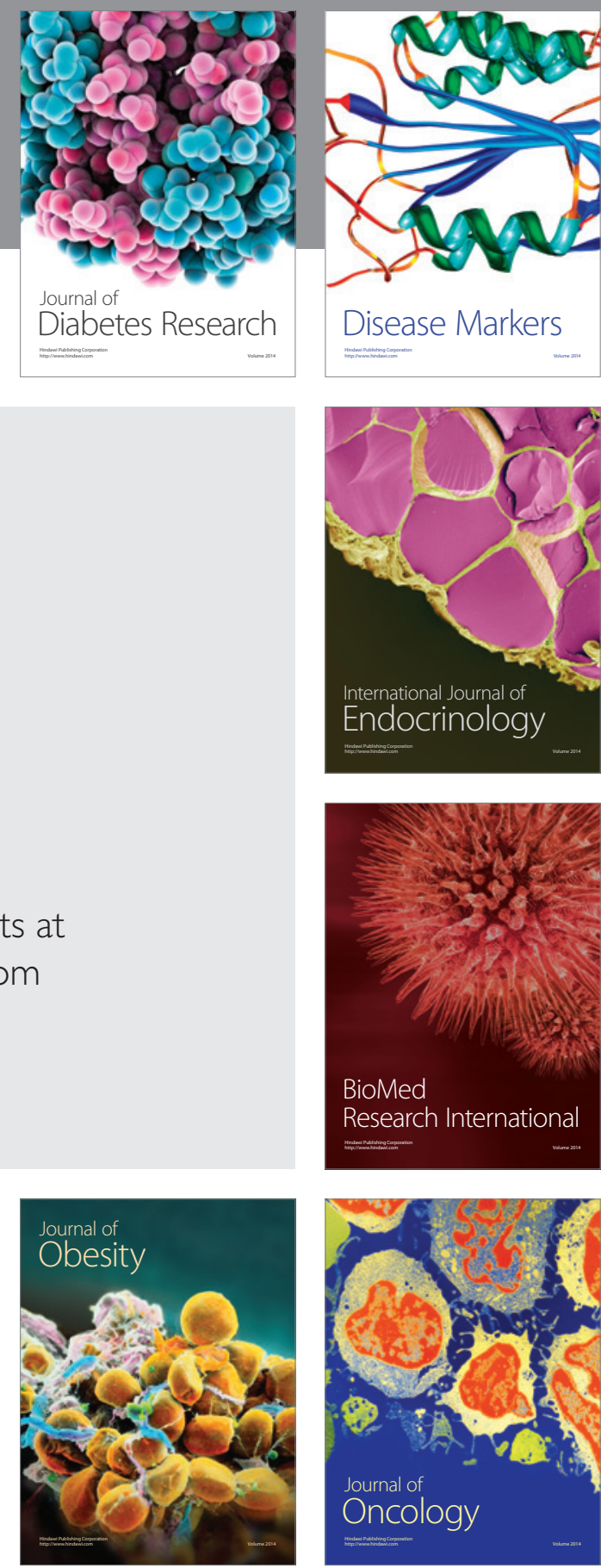

Disease Markers
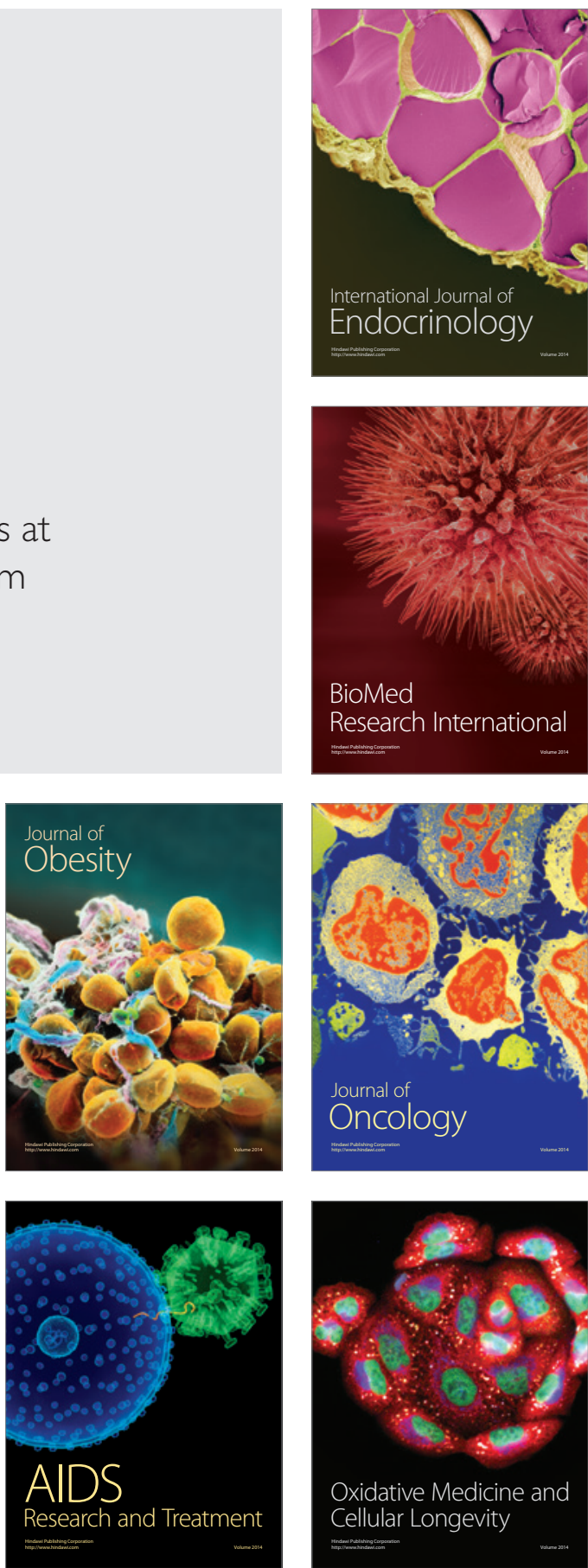